\title{
The role of differential diagnosis in intravascular papillary endothelial hyperplasia of the sinonasal cavity mimicking angiosarcoma: A case report
}

\author{
VITTORIO D'AGUANNO ${ }^{1}$, MASSIMO RALLI² ${ }^{2}$ ARMANDO DE VIRGILIO ${ }^{3}$, \\ ANTONIO GRECO $^{1}$ and MARCO DE VINCENTIIS ${ }^{1}$ \\ Departments of ${ }^{1}$ Sense Organs and ${ }^{2}$ Oral and Maxillofacial Sciences, Sapienza University of Rome, \\ Rome I-00186; ${ }^{3}$ Humanitas Clinical and Research Center, Milan I-20089, Italy \\ Received January 4, 2018; Accepted October 25, 2018
}

DOI: $10.3892 / \mathrm{ol} .2018 .9717$

\begin{abstract}
Intravascular papillary endothelial hyperplasia (IPEH), also known as Masson's tumour, is an unusual vascular lesion characterized by the proliferation of endothelial cells. The principal significance of IPEH is its clinical and radiological resemblance to angiosarcoma, resulting in a potential misdiagnosis and overtreatment. The aim of the present study is to report a rare case of IPEH of the right maxillary sinus, focusing on the radiological and histopathological features of the neoplasm and their role in the differential diagnosis with angiosarcoma. Herein we present a case of a 67-year-old woman with history of right-sided nasal obstruction. A computed tomography scan indicated opacity in the right maxillary sinus and significant bony erosion, and an magnetic resonance imaging revealed a highly vascularized polylobed neoplasm, suggesting a malignant neoplasm. The mass was removed surgically through a combined open and endoscopic approach. A perioperative biopsy excluded malignancy and histological examination was consistent with IPEH. IPEH in the sinonasal cavity is an extremely rare condition in which differential diagnosis serves a central role due to its close clinical and radiological resemblance to angiosarcoma. Awareness of IPEH is recommended for a correct diagnosis, and in order to avoid unnecessarily aggressive treatment.
\end{abstract}

\section{Introduction}

Intravascular papillary endothelial hyperplasia (IPEH), also known as Masson's tumour, is a benign, vascular lesion, characterized by reactive proliferation of the endothelial cells

Correspondence to: Dr Massimo Ralli, Department of Oral and Maxillofacial Sciences, Sapienza University of Rome, 155 Viale del Policlinico, Rome I-00186, Italy

E-mail: massimo.ralli@uniroma1.it

Key words: intravascular papillary endothelial hyperplasia, Masson's tumour, angiosarcoma, sinonasal cavity, differential diagnosis that arise in organizing thrombus (1). This condition was first described by the French pathologist Pierre Masson in 1923 (2). IPEH is typically confined to the lumen of thrombosed pre-existing vessels or vascular malformations (3); any vessel in the body may be involved (4).

Diagnosis of IPEH is often challenging due to its close clinical, radiological and histopathological resemblance to angiosarcoma, especially in the case of endothelial proliferation (5). Thus, correct preoperative diagnosis is essential to prevent aggressive treatment and performing a perioperative biopsy is often necessary to exclude features of malignancy, although considerable bleeding may occur (6).

The occurrence of IPEH in the sinonasal cavity is extremely rare, with only four cases reported in the literature (6-8). In this paper, we report the case of a 67-year-old woman with an IPEH of the right maxillary sinus, focusing on the radiological and histopathological features of the neoplasm and their role in the differential diagnosis with angiosarcoma.

\section{Case report}

A 67-year-old woman was admitted to the Otolaryngology Department of the Policlinico Umberto I, Sapienza University of Rome, in early 2014 with a 6-month history of right-sided nasal obstruction and intermittent aching on the right cheek with rhinorrhoea and postnasal discharge, non-responding to antibiotic and corticosteroid therapy.

After admission, the patient underwent a complete otolaryngology examination. Facial inspection showed oedema of zygomatic region with normal epidermis. Palpation of right infraorbital region produced severe pain. Anterior rhinoscopy indicated a pseudo-polyposis mass originating from the right middle turbinate, with mucous draining from middle meatal region.

Computed tomography (CT) scan without contrast showed a complete opacity within the right maxillary sinus with significant evidence of bony erosion especially in the medial wall (Fig. 1). Magnetic resonance imaging (MRI) with contrast showed a polylobate neoplasm of $47 \times 52 \times 47 \mathrm{~mm}$ in the maxillary sinus, with inhomogeneous high intensity in the T2 weighed images, hypointense in $\mathrm{T} 1$ with high-intensity spots compatible with blood content (Fig. 2). The neoplasm showed 
contrast enhancement with irregular morphology, extending to the medial wall and to the orbital floor with focal signs of bony erosion. Angiography showed a significant vascularization of the mass, thus a super-selective embolization was performed in the terminal branch of the internal maxillary artery to avoid intra-operative haemorrhage.

The clinical and radiologic features in the present case suggested a malignant neoplasm. The patient underwent surgery through a combined approach, open and endoscopic, to allow better visualization and safer eradication of the disease. Perioperative biopsy excluded features of malignancy, and the mass was completely removed (Fig. 3). Diagnosis of IPEH was confirmed by postoperative histological examination. The tissue that macroscopically appeared as a reddish tissue with rubbery texture, revealed a dishomogeneous thrombotic soft tissue characterized by an arborizing network of small blood vessels anastomosed among the tissue, with deposition of matrix and collagen; H\&E sections showed dilated vessels with the presence of organized thrombi. No atypia or mitotic activity were shown in the epithelium (Fig. 4).

Follow-up CT scan performed 6 months after surgery showed a complete eradication of the mass. No recurrence was found after 36 months.

The patient provided written informed consent for the publication of any associated data and accompanying images.

\section{Discussion}

Intravascular papillary endothelial hyperplasia (IPEH) is a plentiful, usually intravascular, benign endothelial proliferation that may mimic angiosarcoma (1).

Pathogenesis of IPEH is still unclear. Masson first described an intravascular endothelial proliferation associated with a haemorrhoidal lesion in the infected veins of a 68 -year-old man and suggested that the endothelial proliferation may be a true neoplasm (2). Henschen (9) reported multiple examples of IPEH in vessels throughout the body and proposed that these neoplasms may be the result of an unusual reactive reparative response of endothelial cells to inflammation and stasis within the vascular bed. Currently, this lesion is considered an unusual form of thrombus organization with exuberant proliferation $(10,11)$ forming as a matrix for the ingrowth of the papillary structure. Hashimoto et al described three forms of IPEH: A pure or primary form, which occurs within a dilated vascular space; a secondary or mixed form, which occurs within a pre-existing vascular lesion such as cavernous and capillary haemangiomas, pyogenic granulomas, arteriovenous malformations, lymphangiomas, and vascular hamartomas; and a third or extravascular form, which arises in a hematoma (11).

A literature review including 314 cases of IPEH (12) showed that $56 \%$ of cases were primary, $40 \%$ secondary form, and $4 \%$ were an extravascular form. The head and neck involvement was found in about one third of the cases, most frequent localizations were dermis, lip, tongue and buccal mucosa. Rare locations included the thyroid, orbit, parotid gland, masseter muscle, nose, sinus, mandible, pharynx and central nervous system (13).

IPEH is a rare condition in sinonasal region: To the best of the authors' knowledge, only three case of IPEH have been reported in the maxillary sinus (6-8), and only one in the ethmoid sinus (13).

Although similar cases have been reported, in our case the presence of significant evidence of bony erosion especially in the medial wall of maxillary sinus might be related to a malignant condition.

Differential diagnosis plays a central role to exclude other vascular neoplasm such as angiosarcoma, hemangiomas, and hemangiopericytoma; a correct diagnosis is therefore essential to prevent radical overtreatment and possible adjuvant systemic treatment that should be otherwise indicated for malignant neoplasms. Radiological characteristics are often unspecific to diagnose IPEH. CT scan of sinonasal IPEH usually demonstrates an expansible contrast-enhanced mass, which could erode the surrounding bone structures. On MRI, T2-weighted images may show a hyperintense mass, with multiple septa and hypointense peripheral rim with strong and heterogeneous enhancement after injection of gadolinium. T1 weighted images show an isointense to slightly hypo-intense mass, with some hyperintense spots within the mass compartment.

Pathological evaluation is necessary to achieve a definitive diagnosis of IPEH. The most important finding is the formation of endothelium-lined papillary structures confined within vascular spaces. The papillary structures are covered by a single layer of swollen or plump endothelial cells around a core of fibrous connective tissue. The papillae might fuse, resulting in a network of vascular channels. Large amounts of thrombi of different sizes might be detected within lesions, sometimes showing various stages of organization $(7,11)$. The microscopic resemblance of IPEH to angiosarcoma and possible misinterpretation is the major pathological significance. Close similarities include the formation of multiple papillae and predominant hypertrophy of endothelia cells, which may show suspicious nuclear atypia. Histopathologically, there are several important distinguishing features that are useful in ruling out a diagnosis of angiosarcoma: i) The endothelial proliferation is confined to the vascular spaces (angiosarcomas are rarely intravascular and tend to invade surrounding tissues); ii) most of the papillary structures are associated with thrombi; iii) lack of necrosis and solid areas; and iv) absence of mitotic figures and cellular pleomorphism (7).

Although histological findings may be sufficient for a diagnosis of IPEH, immunohistochemistry has gained increasing importance for further confirmation of IPEH diagnosis. IPEH is typically positive for CD31, CD34 and SMA (14). It also shows positive staining for ferritin, type VIII collagen, and vimentin. CD31 and CD34 antigens are not endothelial cell specific, but are expressed by vascular endothelium, especially in pathological conditions (15). Akdur et al investigated the morphological and immunohistochemical staining characteristics of 10 IPEH cases focusing on CD31, CD34, FVIII, type IV collagen, SMA, MSA, CD105 and Ki-67 markers. The authors observed CD31 and CD34 staining in all 10 cases (100\%), thus suggesting that the use of immunohistochemical markers in IPEH diagnosis may enhance the ability to differentiate between vascular lesions (16). In the present case, immunohistochemistry has not been performed.

Several surgical approaches have been proposed for IPEH. Endoscopic management has been reported in a few studies $(6,8)$ and could be both diagnostic and curative $(8,17)$. 

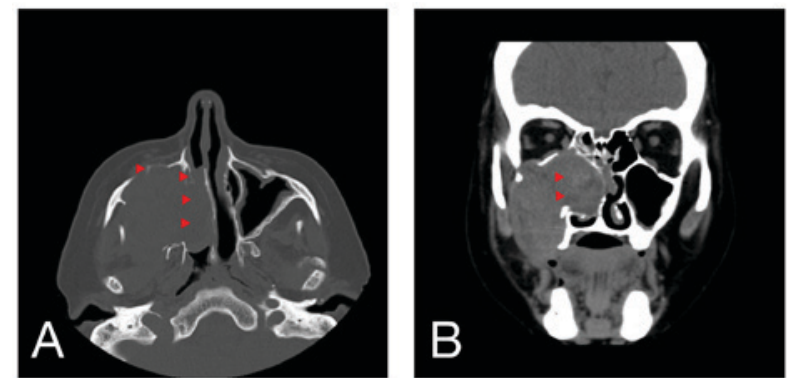

Figure 1. Computed tomography (CT) scan without iodate contrast, in (A) axial and (B) coronal plane, showing a complete opacity within the right maxillary sinus and significant evidence of bony erosion especially in the medial wall (red arrows)
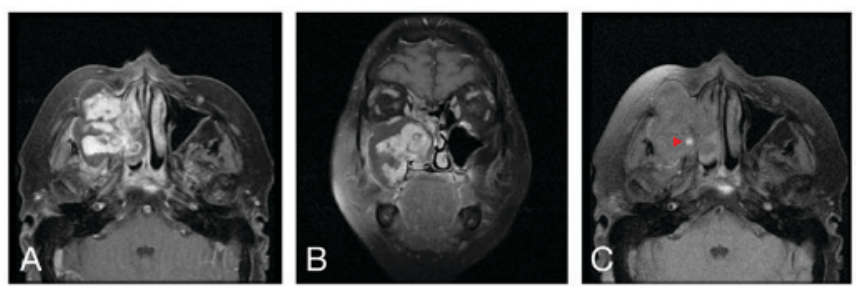

Figure 2. Contrast-enhanced MRI. T2 weighted images in (A) axial and (B) coronal plane showed a large mass measuring $47 \times 52 \times 47 \mathrm{~mm}$. (C) $\mathrm{T} 1$ weighted scan showed a hypointense, polylobate neoplasm with high-intensity spots, compatible with hemosiderin deposits (red arrow). The mass caused deformation and thinning of the sinus walls with bony erosion and significant signs of discontinuity. The medial wall was no longer recognizable, with erosion of the middle turbinate and the root of the lower turbinate. MRI, magnetic resonance imaging.

Open approach via Caldwell-Luc procedure has been described to approach the maxillary sinus with a complete eradication of the disease (7) or via a Weber-Ferguson incision for a case of IPEH in the ethmoid bone (13). In our case, a combined approach, open and endoscopic, has been performed to allow a better visualization and a safer eradication of the disease.

Recurrence of IPEH following incomplete excision of the lesion was documented $(4,17)$ as a result of poor exposure and visualization of the lesion, although no reported instances of metastases had been reported.

IPEH is an unusual vascular lesion characterized by a proliferation of endothelial cells around a thrombus that is undergoing organization. IPEH in the sinonasal cavity is extremely rare and differential diagnosis is often challenging due to its close clinical, radiological and histopathological resemblance to angiosarcoma. Definitive diagnosis of IPEH requires histopathological examination and is essential to prevent aggressive treatment. Surgical excision is the treatment of choice in a case of IPEH.

\section{Acknowledgements}

The authors acknowledge the contributions of Dr Martina Di Brino (Department of Radiology, Sapienza University, Rome, Italy) who carefully reviewed the CT and MRI images.

\section{Funding}

No funding was received.

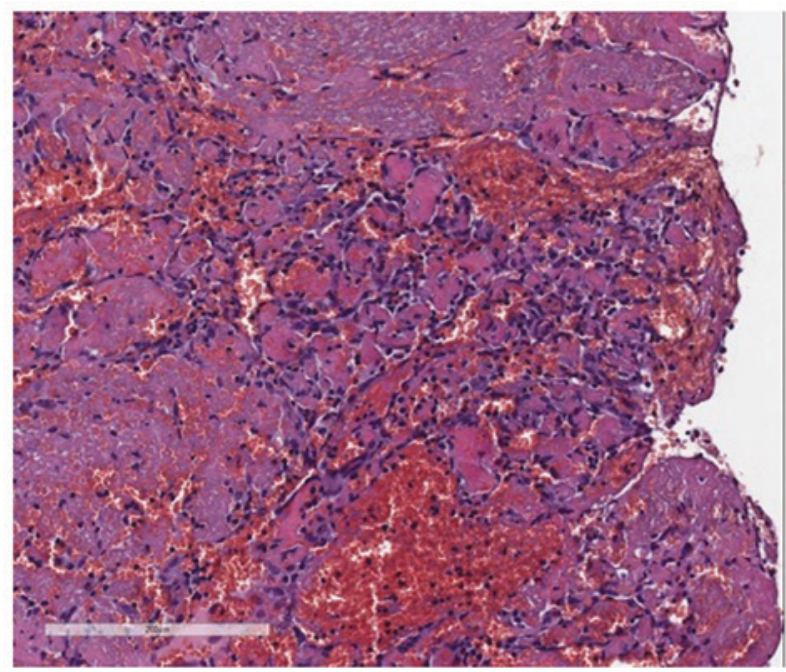

Figure 3. Histological examination of the specimen (hematoxylin and eosin staining; at x100 magnification) revealed a dishomogeneous thrombotic soft tissue characterized by an arborizing network of small blood vessels anastomosed among the tissue, with deposition of matrix and collagen, and the presence of organized thrombi within dilated vessels. No atypia or mitotic activity were shown in the epithelium.

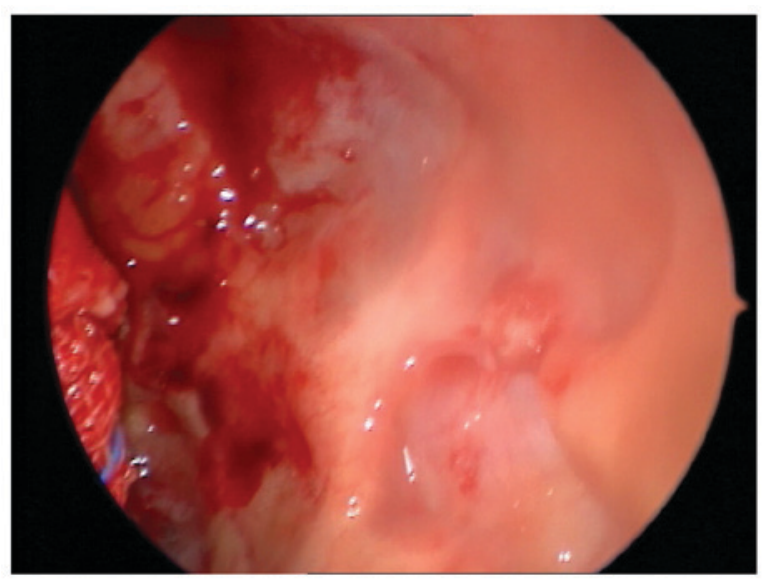

Figure 4. Intraoperative image of maxillary sinus after removing the mass. Posterior wall sinus mucosa is quite normal and no alteration can be seen.

\section{Availability of data and materials}

The datasets used and/or analyzed during the current study are available from the corresponding author on reasonable request.

\section{Authors' contributions}

VD and MR wrote the manuscript. VD, MR, ADV, AG and MDV contributed to the treatment of the patient. All authors read and approved the manuscript.

\section{Ethics approval and consent to participate}

The study and procedures performed were in accordance with the ethical standards of the ethics committee on human experimentation of the Department of Sense Organs of the Sapienza University of Rome, that specifically approved this study, and with the Helsinki Declaration. Written informed consent for 
the publication of any associated data and accompanying images was obtained from the patients or their guardians.

\section{Patient consent for publication}

The patient provided written informed consent for the publication of this study.

\section{Competing interests}

The authors declare that they have no competing interests.

\section{References}

1. Kuo T, Sayers CP and Rosai J: Masson's 'vegetant intravascular hemangioendothelioma:' A lesion often mistaken for angiosarcoma: Study of seventeen cases located in the skin and soft tissues. Cancer 38: 1227-1236, 1976.

2. Masson P: Hèmangioendothèiome vegetant intravascularie. Bull Soc Anat 93: 517-532, 1923.

3. Hong SS, Lee JS, Lee KH, Lee IC, Jun SY, Song KS, Seo JB and Lim TH: Intravascular papillary endothelial hyperplasia of the lung. J Comput Assist Tomogr 26: 362-364, 2002.

4. Stevens DJ: Papillary endothelial hyperplasia in the nose. J Laryngol Otol 102: 935-937, 1988.

5. Enzinger $\mathrm{F}$ and Weiss S: Soft Tissue Tumors. 2nd Edition. CV Mosby Co., St. Louis, pp745-756, 1988.

6. Wang ZH, Hsin CH, Chen SY, Lo CY and Cheng PW: Sinonasal intravascular papillary endothelial hyperplasia successfully treated by endoscopic excision: A case report and review of the literature. Auris Nasus Larynx 36: 363-366, 2009.
7. Stern Y, Braslavsky D, Segal K, Shpitzer T and Abraham A: Intravascular papillary endothelial hyperplasia in the maxillary sinus. A benign lesion that may be mistaken for angiosarcoma. Arch Otolaryngol Head Neck Surg 117: 1182-1184, 1991.

8. Lancaster JL, Alderson DJ, Sherman IW and Clark AH: Papillary endothelial hyperplasia (Masson's tumour) of the maxillary sinus. J Laryngol Otol 112: 500-502, 1998.

9. Henschen P: L'endovasculite proliferante thrombopoietique dans la lesion vasculaire locale. Ann Anat Pathol 9: 113-121, 1932.

10. Clearkin KP and Enzinger FM: Intravascular papillary endothelial hyperplasia. Arch Pathol Lab Med 100: 441-444, 1976.

11. Hashimoto H, Daimaru Y and Enjoji M: Intravascular papillary endothelial hyperplasia: A clinicopathologic study of 91 cases. Am J Dermatopathol 5: 539-546, 1983.

12. Pins MR, Rosenthal DI, Springfield DS and Rosenberg AE: Florid extravascular papillary endothelial hyperplasia (Masson's pseudoangiosarcoma) presenting as a soft-tissue sarcoma. Arch Pathol Lab Med 117: 259-263, 1993.

13. Moon WS, Chung GH and Hong KH: Intravascular papillary endothelial hyperplasia in a vascular lesion of the paranasal sinus. Arch Pathol Lab Med 124: 1224-1227, 2000.

14. Levere SM, Barsky SH and Meals RA: Intravascular papillary endothelial hyperplasia: A neoplastic 'actor' representing an exaggerated attempt at recanalization mediated by basic fibroblast growth factor. J Hand Surg Am 19: 559-564, 1994.

15. Russell Jones R, Orchard G, Zelger B and Wilson Jones E: Immunostaining for CD31 and CD34 in Kaposi sarcoma. J Clin Pathol 48: 1011-1016, 1995.

16. Akdur NC, Donmez M, Gozel S, Ustun H and Hucumenoglu S: Intravascular papillary endothelial hyperplasia: Histomorphological and immunohistochemical features. Diagn Pathol 8: 167, 2013.

17. Safneck JR, Alguacil-Garcia A and Dort JC: Intranasal papillary endothelial hyperplasia. Otolaryngol Head Neck Surg 113: 766-770, 1995 